\title{
INFLUENCIA DE LOS PARÁMETROS METEOROLÓGICOS EN LA CONTAMINACIÓN DEL AIRE EN LA CIUDAD DE SANTANDER \\ A. Martínez-Fernández ${ }^{1}$, S. ${ }^{2}$ Sainz-Villegas, ${ }^{3}$ C. Blanco-Alegre, ${ }^{4}$ F. Oduber, A.I. Calvo, ${ }^{5} \mathrm{~A}$. Castro, ${ }^{6} \mathrm{R}$. Fraile \\ Departamento de Física, IMARENAB Universidad de León, 24071 León, España \\ 1amartf20@estudiantes.unileon.es; ${ }^{2}$ ssainv00@estudiantes.unileon.es; cblaa@unileon.es; 33fodup@unileon.es; ${ }^{4}$ aicalg@unileon.es; ${ }^{5}$ acasi@unileon.es; ${ }^{6}$ rfral@unileon.es
}

\section{Resumen}

Con este trabajo se pretende calcular, para cada tipo de tiempo, la concentración media de contaminantes presentes en la atmósfera y buscar las correlaciones significativas entre la concentración de dichos contaminantes y algunos parámetros meteorológicos en la ciudad de Santander. Para analizar las posibles diferencias significativas, se han aplicado tests no paramétricos.

Estudios recientes han relacionado la exposición a contaminantes atmosféricos, en especial a material particulado, con enfermedades respiratorias y cardiovasculares, entre las que encontramos cáncer bronquial, enfisemas pulmonares, trombosis, coágulos e infartos que, en algunos casos, han llegado a causar la muerte (Rückerl et al., 2011). De los 3 millones de muertes debidas a la contaminación atmosférica que han sido estimadas en el mundo, alrededor de 193.000 han ocurrido en Europa y 7000 en España (Ortiz et al., 2017).

En España la contaminación atmosférica es un problema emergente, sobre todo en las ciudades más pobladas, lo que ha llevado a adoptar medidas para reducir las concentraciones de los contaminantes presentes en el aire, principalmente provenientes de la industria y del transporte.

Los eventos más críticos de contaminación atmosférica se asocian fundamentalmente a situaciones anticiclónicas e inversiones térmicas, que dificultan considerablemente la dispersión atmosférica, creando así una cúpula de contaminantes sobre la zona.

El objetivo principal del presente trabajo es calcular para cada tipo de tiempo la concentración media de contaminantes presentes en la atmósfera y buscar las correlaciones significativas entre la concentración de los contaminantes y los parámetros meteorológicos obtenidos de los radiosondeos (diferenciando entre los tres tipos de estaciones de calidad del aire utilizadas, tráfico, industrial y urbana de fondo). Se ha aplicado el test no paramétrico de Kruskal-Wallis para analizar las diferencias significativas entre las diferentes estaciones de los contaminantes medidos.

El gran volumen de tráfico e industria de la ciudad de Santander y la falta de estudios en la zona hacen de esta ciudad un área de estudio ideal para evaluar su calidad del aire. El presente trabajo pretende buscar relaciones entre la concentración de los contaminantes atmosféricos y las condiciones atmosféricas durante el año 2016. 
Para llevar a cabo el estudio se han empleado:

(i) la concentración de diferentes contaminantes atmosféricos cuyos datos son recogidos por las estaciones de la Red de Calidad del Aire de Cantabria, y que están disponibles en https://www.airecantabria.com/. Dichas estaciones se encuentran localizadas en la Calle Tetuán (estación urbana de fondo), la Plaza de las Estaciones (estación de tráfico) y el Astillero en la Calle Ballestas (estación de industria), como se muestra en la Figura 1. En la tabla 1 se presentan los contaminantes muestreados así como las técnicas de medida utilizadas;

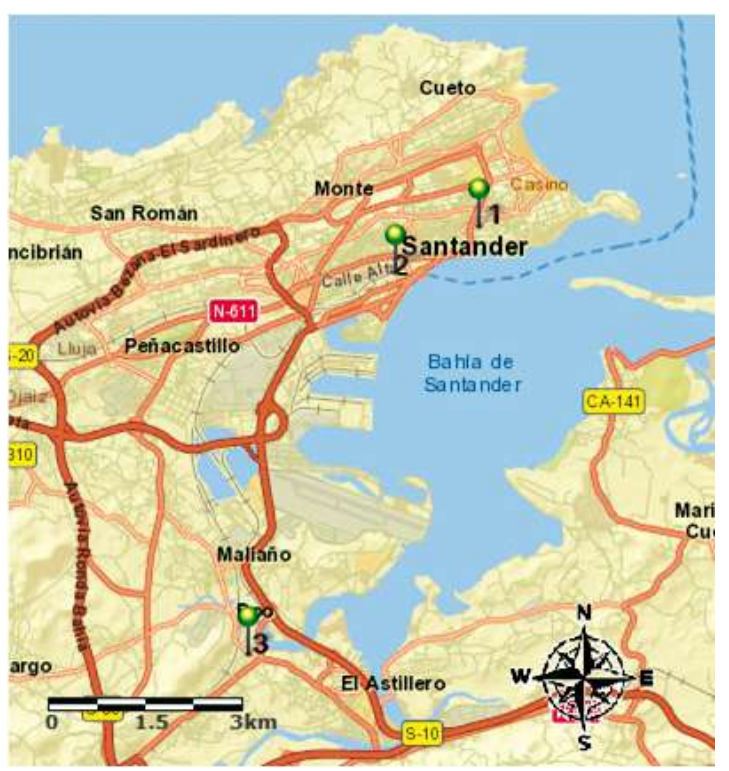

(ii) un total de 54 parámetros meteorológicos obtenidos a partir de los radiosondeos diarios descargados de la página OGIMET (http:// www.ogimet.com/sond.phtml). Han sido extraídos a partir del tratamiento de los datos de los radiosondeos mediante el programa informático RAOB.

Fig. 1.- Localización de las estaciones: Calle Tetuán (urbana de fondo) (punto 1), Plaza de las Estaciones (tráfico) (punto 2) y Astillero (Calle Ballestas) (industria) (punto 3).

\section{CONTAMINANTE}

$\begin{array}{lccc}\text { Dióxido de azufre } & \mathrm{SO} 2 & \mu \mathrm{g} / \mathrm{m} 3 & \text { Fluorescencia UV } \\ \text { Monóxido de carbono } & \mathrm{CO} & \mu \mathrm{g} / \mathrm{m} 3 & \text { Absorción infrarroja } \\ \text { Monóxido de nitrógeno } & \mathrm{NO} & \mu \mathrm{g} / \mathrm{m} 3 & \text { Quimioluminiscencia } \\ \text { Dióxido de nitrógeno } & \mathrm{NO} 2 & \mu \mathrm{g} / \mathrm{m} 3 & \text { Quimioluminiscencia } \\ \text { Partículas }<10 \mu \mathrm{m} & \mathrm{PM} 10 & \mu \mathrm{g} / \mathrm{m} 3 & \text { Microbalanza } \\ \text { Ozono } & \mathrm{O} 3 & \mu \mathrm{g} / \mathrm{m} 3 & \text { Absorción ultravioleta } \\ \text { Tolueno } & \mathrm{TOL} & \mu \mathrm{g} / \mathrm{m} 3 & \text { Cromatografía de gases } \\ \text { Benzeno } & \mathrm{BEN} & \mu \mathrm{g} / \mathrm{m} 3 & \text { Cromatografía de gases }\end{array}$

Tabla 1.- Listado de contaminantes atmosféricos empleados en el estudio y técnicas de medida. 


\section{SEVERE WEATHER}

Temperatura punto de rocío en superficie $\left({ }^{\circ} \mathrm{C}\right)$

Temperatura punto de rocío en superficie a $850 \mathrm{mb}\left({ }^{\circ} \mathrm{C}\right)$

Velocidad del viento a $850 \mathrm{mb}$ (kt)

Velocidad del viento a $500 \mathrm{mb}$ (kt)

Velocidad del viento a $200 \mathrm{mb}$ (kt)

LFC - Nivel de convección libre (hPa)

$\mathrm{LI}$ - Îndice de elevación $\left({ }^{\circ} \mathrm{C}\right)$

$\mathrm{SI}$ - Índice de Showalter $\left({ }^{\circ} \mathrm{C}\right)$

$\mathrm{TI}$ - Índice de Thompson $\left({ }^{\circ} \mathrm{C}\right)$

$\mathrm{JI}$ - Índice de Jefferson $\left({ }^{\circ} \mathrm{C}\right)$

Índice $\mathrm{K}\left({ }^{\circ} \mathrm{C}\right)$

Índice TT (Total Totals) $\left({ }^{\circ} \mathrm{C}\right)$

SWEAT - Índice de amenaza de tiempo severo

CAPE Total - Energía potencial convectiva disponible (J/kg)

CAPE 0-x km, AGL (J/kg)

$\mathrm{srH}$ - Helicidad relativa a la tormenta $(0-3 \mathrm{~km})(\mathrm{m} 2 \mathrm{~s}-2)$

EHI - Índice de Energía - Helicidad

VGP - Parámetro de generación de vorticidad ( $\mathrm{m} \mathrm{s}$-2)

CAP Strength $\left({ }^{\circ} \mathrm{C}\right)$

BRN - No Richardson global.

BRN - Cizalladura del No de Richardson global (m s-1)

WBZ - Altura del cero de termómetro húmedo (ft,AGL)

Tamaño probable de granizo (in)

WINDEX (kn)

T2 Gust (kn)

Índice $\mathrm{KO}\left({ }^{\circ} \mathrm{C}\right)$

Índice Boyden

Índice $\mathrm{S}\left({ }^{\circ} \mathrm{C}\right)$

\section{DATA ANALYSES}

Presión superficie $(\mathrm{hPa})$

Temperatura superficie $\left({ }^{\circ} \mathrm{C}\right)$

Temperatura de punto de rocío en superficie $\left({ }^{\circ} \mathrm{C}\right)$

Altura 1000 mb (m)

Dirección (grados)

Velocidad (kn)

Tc - Temperatura convectiva $\left({ }^{\circ} \mathrm{C}\right)$

Altura Tropopausa (m)

Altura a $-20^{\circ} \mathrm{C}(\mathrm{m})$

Altura a $-10{ }^{\circ} \mathrm{C}(\mathrm{m})$

Altura a $-5^{\circ} \mathrm{C}(\mathrm{m})$

Altura a $0^{\circ} \mathrm{C}(\mathrm{m})$

Altura inversión (m)

Tipo inversión

ccl-EL - Nivel de equilibrio basado en CCL (m)

Ifc-EL - Nivel de equilibrio basado en LFC (m)

$$
\begin{aligned}
& \mathrm{LFC}(\mathrm{m}) \\
& \mathrm{CCL}(\mathrm{m})
\end{aligned}
$$$$
\mathrm{LCL}(\mathrm{m})
$$

$1000-500 \mathrm{mb}(\mathrm{m})$

$1000-700 \mathrm{mb}(\mathrm{m})$

$1000-850 \mathrm{mb}(\mathrm{m})$

$850-500 \mathrm{mb}(\mathrm{m})$

$850-700 \mathrm{mb}(\mathrm{m})$

$700-500 \mathrm{mb}(\mathrm{m})$

600- $400 \mathrm{mb}(\mathrm{m})$

Tabla 2.- Lista de parámetros extraídos con el programa RAOB y unidades.

\section{TIPOS ANTICICLONICOS DIRECCIONALES PUROS}

A

ANE anticiclónico-noreste $\mathrm{NE}$

$\mathrm{AE}$ anticiclónico-este $\mathrm{E}$

ASE anticiclónico-sureste SE

AS anticiclónico-sur $S$

ASW anticiclónico-suroeste SW

AW anticiclónico-oeste W

ANW anticiclónico-noroeste NW

AN anticiclónico-norte $\mathrm{N}$
C

$\begin{array}{cc}\text { noreste } & \text { CNE } \\ \text { este } & \text { CE } \\ \text { sureste } & \text { CSE } \\ \text { sur } & \text { CS }\end{array}$

suroeste CSW

oeste CW

noroeste $\mathrm{CNW}$

norte $\quad \mathrm{CN}$
TIPOS CICLONICOS

ciclónico

ciclónico-noreste ciclónico-este

ciclónico-sureste ciclónico-sur

ciclónico-suroeste ciclónico-oeste

ciclónico-noroeste ciclónico-norte

Tabla 3.- Tipos de tiempo según la clasificación de Lamb. 
Adicionalmente se ha realizado un estudio de los tipos de tiempo, de acuerdo con la clasificación de Lamb (1972). La clasificación de tipos de tiempo efectuada tiene un carácter objetivo y establece cada tipo de tiempo en función de seis variables basadas en la dirección y vorticidad del viento geostrófico, que son calculadas según los valores de presión atmosférica, estimados diariamente a nivel del mar en 16 puntos situados sobre el entorno de la península ibérica. Los valores de la presión en superficie se obtuvieron del IRI (International Research Institute for Climate Prediction) en http://www.cdc. noaa.gov/cdc/data.ncep.reanalysis.pressure.html. Así, los tipos de tiempo se clasifican en 9 tipos anticiclónicos, 8 direccionales puros y 9 ciclónicos (tabla 3).

Los tipos de tiempo más habituales durante el año 2016 fueron el anticiclónico (A), y los direccionales puros NE y $\mathrm{N}$ en un $18 \%, 11 \%$ y $10 \%$ de los días respectivamente (figura 2).

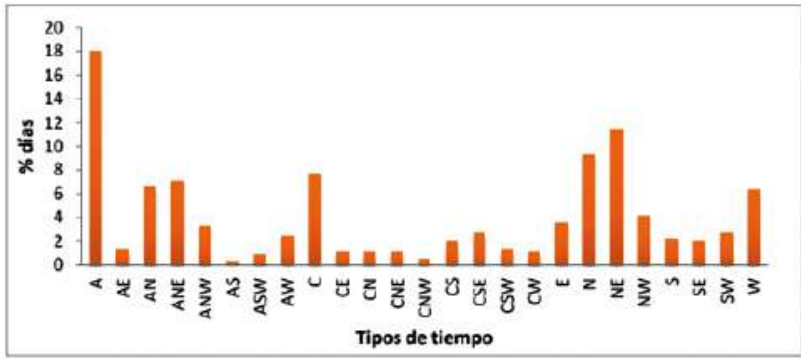

Fig. 2.- Porcentaje de tipos de tiempo en el año 2016.

\section{Agradecimientos}

Este trabajo ha sido parcialmente financiado por el Ministerio de Economía y Competitividad (TEC2014-57821-R), la Universidad de León (Programa Propio 2015/00054/001) y el proyecto AERORAIN (Ministerio de Economía y Competitividad, CGL2014-52556-R, cofinanciado con fondos FEDER). F. Oduber disfruta de un contrato BES-2015-074473 del Ministerio de Economía y Competitividad. C. Blanco-Alegre disfruta de un contrato FPU16-05764 del Ministerio de Educación, Cultura y Deporte.

\section{Referencias}

- Lamb, H. 1972. British Isles Weather types and a register of daily sequence of circulation patterns. Geophysical Memoir, 116, 85.

- Ortiz, C., Linares, C., Carmona, R., Díaz, J. 2017. Evaluation of short-term mortality attributable to particulate matter pollution in Spain, Environ. Pollution. http://dx.doi.org/10.1016/j. envpol.2017.02.037

- Rückerl, R., Schneider A., Breitner S., Cyrys J., Peters A.. 2011. Health effects of particulate air pollution: a review of epidemiological evidence. Inhal. Toxicol. 23 (10), 555-592. 